\title{
Evolução da mortalidade por homicídio no Estado da Bahia, Brasil, no período de 1996 a 2010
}

The evolution of mortality by homicide in the State of Bahia in the period from 1996 to 2010

${ }^{1}$ Centro Latino-Americano de Estudos de Violência e Saúde Jorge Careli, Escola Nacional de Saúde Pública, Fundação Oswaldo Cruz. Av. Brasil 4036/700,

Manguinhos. 21.040-361

Rio de Janeiro RJ

Brasil.tiagosouza@

posgrad.ensp.fiocruz.br
Abstract An ecological study was conducted, the objective of which was to describe the evolution of homicide rates for residents of the State of $\mathrm{Ba}$ hia, Brazil, and its nine health macroregions (MRS) in the period from 1996 to 2010. Crude and adjusted data from the Mortality Information System (SIM) and X85-Y09 codes of the Tenth International Statistical Classification of Diseases and Related Health Problems (ICD-10) were analyzed. The number, proportion and homicide mortality rates (TMH) were analyzed. considering the characteristics of the victim and the event. The results revealed a profile of predominantly male mortality, Afro-Brazilian race/ethnicity with little education. The public highways were the main location of occurrence of deaths. There was an increase in TMH in all age groups, though the highest rates were observed in the population of 15 to 39 years of age. The TMH age-standardized rates were higher in the Far South, East, North and South. The conclusion reached was that the killings can be addressed from the loation/ regional realities through strategic and planning of intersectoral actions that take into account the socioeconomic and cultural characteristics.

Key words Mortality, Epidemiological Factors, Temporal Distribution, Homicide
Resumo Foi realizado um estudo ecológico com o objetivo de descrever a evolução da mortalidade por homicídio de residentes no Estado da Bahia, Brasil, e em suas nove macrorregiões de saúde (MRS), no período de 1996 a 2010. Foram empregados dados do Sistema de Informação sobre Mortalidade (SIM), sendo utilizados os eventos com os códigos (X85-Y09) da décima Classificação Estatística Internacional de Doenças e Problemas Relacionados à Saúde (CID-10). Analisou-se o número, a proporção e as taxas de mortalidade por homicídio (TMH) brutas e ajustadas, considerando-se as características da vítima e do evento. Os resultados evidenciaram um perfil de mortalidade preponderante masculino, de raça/ etnia negra e com baixa escolaridade. A via pública foi o principal local de ocorrência dos óbitos. Houve incremento das TMH em todas as faixas etárias, mas as maiores taxas foram observadas na população dos 15 aos 39 anos. As TMH padronizadas por idade foram mais elevadas no Extremo Sul, Leste, Norte e Sul. Conclui-se que os homicídios podem ser enfrentados a partir das realidades locorregionais através de estratégias e planejamento de ações intersetoriais, que levem em consideração as características socioeconômicas e culturais.

Palavras-chave Mortalidade, Fatores epidemiológicos, Distribuição temporal, Homicídio 


\section{Introdução}

A violência é um dos maiores problemas da saúde pública deste século, pela forma como afeta, sobretudo, a população jovem. Como se não bastasse, acarreta prejuízos individuais e coletivos, impacta a morbidade e a mortalidade da população e não se restringe apenas ao setor saúde, mas exige esforços de diversos outros como, educação, economia, política, justiça, assistência social, e muitos mais ${ }^{1,2}$. Por esse motivo, tornouse alvo das intervenções da saúde pública no Brasil, no que se refere à atenção às vítimas e à prevenção desses eventos. Para isso, também são necessárias políticas públicas que possibilitem ações intersetoriais organizadas ${ }^{3,4}$. Neste contexto, o setor saúde tem um papel central de integração das ações de prevenção e promoção, juntamente com outros e com a sociedade civil ${ }^{1}$.

Desde que ultrapassou os acidentes de transporte, na última década do século passado, os homicídios vêm preocupando as autoridades e estudiosos do tema, por se colocarem como a primeira causa de morte entre os adolescentes e adultos jovens, no Brasil. Para demonstrar a ascensão do homicídio, como uma das principais causas de óbito nessa parcela da população, Peres et al..$^{5}$ destacaram que o coeficiente de mortalidade por homicídio no Brasil, na população de 0 a 19 anos, apresentou um incremento global de $306,3 \%$, no período de 1980 a 2002. Assim, as agressões letais vêm sendo objeto de diversos estudos ${ }^{1,6}$.

O homicídio configura-se como uma expressão da violência interpessoal ${ }^{7}$; é a partir dele que podemos captar a gravidade da situação de violência em um determinado contexto. Além disso, as agressões fatais parecem servir como indicador da violência estrutural e das condições de vida. Estudos nacionais e internacionais mostram que os homens jovens são as principais vítimas dos homicídios, além de serem os principais perpetradores dos mesmos ${ }^{8-10}$. Nesta perspectiva, Souza e Lima ${ }^{8}$ apontam que no Brasil, em 2003, as taxas de homicídio no sexo masculino foram bem superiores às do sexo feminino, com uma razão de risco 12,3 vezes maior (na população total), sendo que na faixa etária, dos 20 aos 24 anos os homens (comparados às mulheres) apresentaram razão de risco ainda mais elevada e taxa de 132,6/100 mil homens.

Historicamente a Bahia não é um Estado que tem exibido altas taxas de homicídios. No entanto, estudos mais recentes sobre a situação de violência no país, têm apontado o fato de Estado e regiões, que outrora mostravam baixos ou médios índices de violência, virem apresentando incrementos preocupantes ${ }^{11}$. Na região Nordeste, o Estado da Bahia evidencia esse crescimento dos homicídios. Sua baixa taxa de mortalidade por essa causa cresceu e desponta entre as dez unidades federativas com maior risco absoluto para violência homicida nos últimos anos ${ }^{11}$. Para se ter uma noção desta mudança, a taxa de homicídio na Bahia, passou de 9,7 no ano de 1998, para 32,9 por 100 mil habitantes em 2008.

Este fenômeno de disseminação dos homicídios de regiões do país mais desenvolvidas socioeconomicamente para regiões menos desenvolvidas, seja em direção ao interior dos estados ou para outros estados da federação, vem sendo denominado de interiorização da violência ${ }^{8,11}$.

A grande maioria dos estudos sobre homicídios aborda as capitais do país, como exemplo, os poucos estudos sobre esse tema na Bahia têm focalizado sua capital, Salvador. Esses estudos abordam aspectos como a mortalidade por causas externas, políticas públicas, anos potenciais de vida perdidos, violência urbana, homicídios entre jovens e desigualdade, entre outros temas ${ }^{12-}$ ${ }^{18}$. Existe, pois, uma carência de conhecimento sobre outras regiões da Bahia, ainda inexploradas, que precisam ser investigadas, no intuito de estabelecer as reais necessidades do Estado, no que se refere a ações específicas e à programação de políticas públicas, nessas áreas.

O homicídio é um importante indicador social e de saúde, e diversos estudos vêm apontando novos padrões de mortalidade no Brasil ${ }^{5,19}$. Assim, considera-se que o estudo desse evento, bem como a definição de políticas públicas e o seu enfrentamento, precisa ser abordado com base no processo de regionalização, que é uma das estratégias fundamentais do Sistema Único de Saúde (SUS) ${ }^{20,21}$. Esse é, portanto, o foco do presente artigo que tem como objetivo descrever a evolução da mortalidade por homicídio no Estado da Bahia e em suas nove macrorregiões de saúde, no período de 1996 a 2010.

\section{Materiais e Métodos}

Trata-se de um estudo ecológico do tipo exploratório, comparativo, no qual se analisou a evolução temporal da mortalidade por homicídio no Estado da Bahia, no período de 1996 a 2010. Este recorte de tempo teve por base o ano de vigência da décima revisão da Classificação Esta- 
tística Internacional de Doenças e Problemas Relacionados à Saúde (CID-10) 22 , que passou a vigorar a partir do ano de 1996; o ano de 2010 foi o último ano para o qual os dados estavam disponíveis no momento da coleta.

Foram selecionados os óbitos dos residentes por Agressões (código X85 a Y09 da CID-10), aqui referidos como homicídios, do Sistema de Informações sobre Mortalidade (SIM), que juntamente com estimativas populacionais estão disponibilizados no sítio eletrônico do Departamento de Informática do Sistema Único de Saúde (DATASUS) do Ministério da Saúde (MS ${ }^{23}$. As informações foram extraídas diretamente do TabNet ${ }^{23}$ Win32 2.7, salvas em planilhas eletrônicas $\left(\right.$ Excel $\left.^{\odot}\right)$ e posteriormente armazenadas em um banco de dados no software Statistical Package for Social Science $\left(\right.$ SPSS $\left.^{\oplus}\right)$ versão 19.0 para processamento, execução dos cálculos, tabulações e análises.

O Estado da Bahia está dividido em nove macrorregiões de saúde (MRS) cujas principais características socioeconômicas e demográficas foram exploradas a fim de caracterizar o contexto territorial onde esses óbitos ocorreram. As características das MRS incluem: população total, proporção da população por sexo, por faixa etária e por raça/cor, número de municípios, área por quilômetro quadrado $\left(\mathrm{km}^{2}\right)$, densidade demográfica (razão entre a população residente e a área), proporção da população com baixa renda, taxa de analfabetismo e de desemprego e índice de desenvolvimento humano (IDH). Essas informações, com exceção do IDH, foram extraídas do Instituto Brasileiro de Geografia e Estatística (IBGE), referentes ao ano de $2010^{24}$. Os dados de área que são disponibilizados por município foram agrupados por macrorregiões e apresentados a partir do total da soma obtida, enquanto que a densidade demográfica foi recalculada (a razão entre a soma da população e a soma das áreas dos municípios que compõem a macrorregião).

A parte da população com baixa renda, anteriormente denominado como "proporção de pobres", constitui-se dos residentes com renda domiciliar mensal per capita inferior a 1/2 salário mínimo, por macrorregião no ano de 2010. A taxa de analfabetismo é o percentual de pessoas com 15 anos de idade e mais que não sabiam ler e escrever pelo menos um bilhete simples, em determinada macrorregião, no ano de 2010. A taxa de desemprego é o percentual da população residente economicamente ativa que se encontra sem trabalho, nas macrorregiões no ano de 2010.
O IDH consiste numa média entre três dimensões básicas: vida longa e saudável (Esperança de vida ao nascer), nível de conhecimento (Taxa de analfabetismo e escolarização) e nível de vida digno (Produto Interno Bruto per capita), o resultado pode variar de 0 a 1 , sendo que quanto mais próximo a 1, melhores os índices de desenvolvimento. O índice foi obtido no sítio eletrônico do Programa das Nações Unidas para o Desenvolvimento (PNUD), conforme os registros dos municípios em 2000, ano mais recente para o qual está acessível ${ }^{25}$. Essa informação também foi agrupada por MRS e apresentada a média dos municípios que compõem cada território em análise.

As macrorregiões de saúde são delimitadas segundo as características das coletividades e das particularidades socioeconômicas dessas áreas territoriais. Essa divisão territorial faz parte do processo de regionalização do SUS, que tem por alicerce a descentralização, princípio organizacional de sua gestão. Cada MRS possui um município que funciona como centro polarizador das ações e serviços de saúde, este é identificado como município polo, e funciona como ponto de referência para as demais cidades distribuídas no seu raio de influência ${ }^{26}$. Utilizar-se desta diretriz, como parte do método, tem por finalidade apontar uma estratégia para a implantação e implementação de políticas públicas intersetoriais, voltadas para a prevenção dos homicídios e controle de seus efeitos.

Analisaram-se ainda as frequências absoluta (número de óbitos) e relativa (proporção de óbitos), segundo as características das vítimas que incluem: sexo, faixa etária, escolaridade, estado civil, raça/cor; e do evento, que são o local de ocorrência do óbito (hospital, domicílio, estabelecimento de saúde, via pública e outros) e a macrorregião de saúde. As idades foram grupadas nas faixas etárias de 0 a 9; 10 a 14; 15 a 19;20 a $29 ; 30$ a $39 ; 40$ a $49 ; 50$ a $59 ; 60$ ou mais anos.

As taxas de mortalidade por homicídio (TMH) foram calculadas para cada ano, de acordo com as macrorregiões de saúde, a faixa etária e o sexo, pela base referencial de 100.000 habitantes. Para analisar a evolução temporal da mortalidade, utilizou-se a variação percentual $(\Delta \%)$ das TMH no início e no final do período, em que $\boldsymbol{T}_{f}$ é a taxa de homicídio do ano final, e $\boldsymbol{T}_{i}$ é a taxa de homicídio do ano inicial.

$$
\Delta \%=\left(\frac{T_{f}-T_{i}}{T_{i}}\right) \times 100
$$


As TMH, segundo as macrorregiões de saúde, foram padronizadas por idade a partir da população do Brasil em 2000, pelo método direto, segundo critério da $\mathrm{OMS}^{27}$, para ajustar o efeito da estrutura etária e viabilizar a comparação livre de distorções entre os índices das diferentes áreas geográficas ${ }^{19,27}$.

Após análise inicial da proporção de homicídios, das taxas e da variação percentual, identificou-se o grupo dos 15 aos 39 anos de idade entre aqueles mais afetados durante o período de estudo. Por isso, detalhou-se a investigação nessa faixa etária, buscando identificar os níveis de vulnerabilidade deste grupo, no ano inicial (1996), no meio do período (2003) e no último ano do estudo (2010).

Este estudo foi aprovado pelo Comitê de Ética em Pesquisa da Escola Nacional de Saúde Pública Sergio Arouca.

\section{Resultados}

A Bahia, com população de 14.016.960 habitantes em 2010, tem 417 municípios agrupados em nove macrorregiões de saúde. As principais características socioeconômicas e demográficas dessas macrorregiões para o ano de 2010 estão apresentadas na Tabela 1. Nela observa-se que $70 \%$ da população total do Estado, encontram-se nas regiões Leste $(31,1 \%)$, Centro-Leste $(15,1 \%)$, Sudoeste $(12,2 \%)$ e Sul $(11,6 \%)$. A MRS Leste, onde está situada a capital, Salvador, possui maior densidade demográfica com $269,2 \mathrm{hab} / \mathrm{km}^{2}$, apresenta maior proporção de pessoas entre 15 e 39 anos e tem $80,8 \%$ da população de raça/cor negra (soma dos pretos e pardos). A proporção da população com baixa renda foi maior nas regiões Centro-Norte $(67,0 \%)$ e Nordeste $(64,9 \%)$, sendo que esta última apresenta ainda, a maior taxa

Tabela 1. Características socioeconômicas e demográficas das Macrorregiões de Saúde. Bahia, Brasil, 2010.

\begin{tabular}{|c|c|c|c|c|}
\hline \multirow[b]{2}{*}{ Característica } & \multicolumn{4}{|c|}{ Macrorregião de Saúde } \\
\hline & $\begin{array}{c}\text { Centro-Leste } \\
\mathrm{N}=2.114 .134\end{array}$ & $\begin{array}{c}\text { Centro-Norte } \\
\mathrm{N}=771.253\end{array}$ & $\begin{array}{l}\text { Extremo Sul } \\
N=760.206\end{array}$ & $\begin{array}{c}\text { Leste } \\
\mathrm{N}=4.353 .829\end{array}$ \\
\hline \multicolumn{5}{|l|}{ População (\%) } \\
\hline Masculina & 49,0 & 50,1 & 50,2 & 47,6 \\
\hline Feminina & 51,0 & 49,9 & 49,8 & 52,4 \\
\hline Número de municípios & 73 & 38 & 21 & 48 \\
\hline Área $\left(\mathrm{km}^{2}\right)$ & $74.023,1$ & $47.541,5$ & $30.667,5$ & $16.171,5$ \\
\hline $\begin{array}{l}\text { Densidade demográfica } \\
\left(\mathrm{hab} / \mathrm{km}^{2}\right)\end{array}$ & 28,6 & 16,2 & 24,8 & 269,2 \\
\hline \multicolumn{5}{|l|}{ Faixa etária (\%) } \\
\hline 0 a 9 anos & 16,6 & 17,6 & 18,2 & 14,1 \\
\hline 10 a 14 anos & 10,0 & 10,3 & 10,4 & 8,5 \\
\hline 15 a 19 anos & 9,8 & 10,0 & 9,6 & 8,6 \\
\hline 20 a 29 anos & 18,2 & 17,3 & 18,2 & 19,9 \\
\hline 30 a 39 anos & 14,7 & 14,1 & 15,0 & 17,4 \\
\hline 40 a 49 anos & 11,4 & 11,3 & 11,5 & 13,3 \\
\hline 50 a 59 anos & 8,0 & 8,1 & 8,1 & 9,3 \\
\hline 60 e mais & 11,2 & 11,3 & 8,9 & 9,1 \\
\hline \multicolumn{5}{|l|}{ Raça/cor (\%) } \\
\hline Branca & 20,6 & 24,8 & 22,6 & 17,5 \\
\hline Preta & 16,8 & 11,3 & 12,1 & 26,9 \\
\hline Amarela & 1,0 & 1,0 & 0,9 & 1,4 \\
\hline Parda & 61,4 & 62,7 & 62,5 & 53,9 \\
\hline Indígena & 0,2 & 0,2 & 2,0 & 0,3 \\
\hline Não informada & 0,0 & 0,0 & 0,0 & 0,0 \\
\hline $\begin{array}{l}\text { Proporção da população } \\
\text { com baixa renda }\end{array}$ & 59,8 & 67,0 & 51,6 & 40,9 \\
\hline Taxa de analfabetismo & 19,1 & 20,5 & 18,5 & 7,2 \\
\hline Taxa de desemprego & 9,5 & 8,9 & 10,7 & 13,0 \\
\hline $\mathrm{IDH}^{*}$ & 0,622 & 0,605 & 0,658 & 0,665 \\
\hline
\end{tabular}


de analfabetismo (23,3\%). As mais altas taxas de desemprego foram observadas, nesta ordem, nas MRS Leste, Sul e Extremo Sul, mas em contrapartida, as regiões Leste e Extremo Sul apresentaram os melhores índices de desenvolvimento humano da Bahia, no ano de 2000, com 0,665 e 0,658, respectivamente (Tabela 1).

Conforme exposto na Tabela 2, durante os 15 anos do período estudado, foram registrados 40.756 homicídios no Estado da Bahia. Grande parte das vítimas $(92,4 \%)$ é do sexo masculino; $78 \%$ dos homicídios concentram-se no estrato dos 15 aos 39 anos e a maior proporção das vítimas tinha até sete anos de estudo ou ensino fundamental incompleto. No entanto, a análise dessa última variável está prejudicada pelo elevado número de registros sem informação ou com informação ignorada (33,0\%), embora o preenchimento sobre escolaridade venha melhorando ao longo dos anos. Em 2010, essa variável foi informada em 78,6\% desses óbitos, porém ela ainda possui o menor nível de completitude dentre todas as variáveis do SIM no estado.

Os solteiros somam a maioria dos óbitos, mais de $85 \%$ dos casos válidos. A raça/cor negra (somados os pretos e pardos) registrou 73,1\% dos homicídios no período; $40,7 \%$ desses óbitos ocorreram em via pública, e em $30,6 \%$ dos casos o local de ocorrência do óbito foi o hospital. Quando se considera a macrorregião de saúde onde residem as vítimas, observa-se que as áreas com maior frequência relativa de homicídio foram a MRS Leste $(45,7 \%)$ e a Sul com $13,7 \%$ (Tabela 2).

A evolução da TMH, segundo faixa etária, mostra que as taxas cresceram em todas elas (Tabela 3). Considerando-se a variação percentual $(\Delta \%)$ entre 1996 e 2010 , os aumentos mais

\begin{tabular}{|c|c|c|c|c|c|}
\hline \multirow[b]{2}{*}{ Característica } & \multicolumn{5}{|c|}{ Macrorregião de Saúde } \\
\hline & $\begin{array}{c}\text { Nordeste } \\
\mathrm{N}=\mathbf{8 1 3 . 2 7 1}\end{array}$ & $\begin{array}{c}\text { Norte } \\
\mathrm{N}=1.001 .075\end{array}$ & $\begin{array}{c}\text { Oeste } \\
\mathrm{N}=\mathbf{8 7 6 . 8 4 3}\end{array}$ & $\begin{array}{c}\text { Sudoeste } \\
\mathrm{N}=1.704 .534\end{array}$ & $\begin{array}{c}\text { Sul } \\
\mathrm{N}=1.621 .761\end{array}$ \\
\hline \multicolumn{6}{|l|}{ População (\%) } \\
\hline Masculina & 49,5 & 49,8 & 51,0 & 49,9 & 49,7 \\
\hline Feminina & 50,5 & 50,2 & 49,0 & 50,1 & 50,3 \\
\hline Número de municípios & 33 & 27 & 37 & 73 & 67 \\
\hline Área $\left(\mathrm{km}^{2}\right)$ & $19.935,4$ & $92.566,2$ & $158.731,6$ & $85.227,8$ & $39.966,2$ \\
\hline $\begin{array}{l}\text { Densidade demográfica } \\
\left(\mathrm{hab} / \mathrm{km}^{2}\right)\end{array}$ & 40,8 & 10,8 & 5,5 & 20,0 & 40,6 \\
\hline \multicolumn{6}{|l|}{ Faixa etária (\%) } \\
\hline 0 a 9 anos & 16,7 & 18,0 & 17,9 & 15,8 & 16,8 \\
\hline 10 a 14 anos & 10,5 & 10,3 & 10,6 & 9,4 & 9,9 \\
\hline 15 a 19 anos & 10,1 & 9,9 & 10,5 & 9,7 & 9,7 \\
\hline 20 a 29 anos & 17,7 & 17,7 & 18,8 & 18,3 & 18,1 \\
\hline 30 a 39 anos & 14,6 & 14,8 & 14,0 & 14,3 & 14,1 \\
\hline 40 a 49 anos & 11,5 & 11,3 & 11,0 & 11,8 & 11,5 \\
\hline 50 a 59 anos & 7,9 & 7,7 & 7,6 & 8,6 & 9,0 \\
\hline 60 e mais & 11,1 & 10,4 & 9,7 & 12,1 & 11,0 \\
\hline \multicolumn{6}{|l|}{ Raça/cor (\%) } \\
\hline Branca & 22,7 & 25,3 & 26,0 & 33,2 & 19,6 \\
\hline Preta & 13,6 & 8,8 & 9,4 & 8,2 & 16,8 \\
\hline Amarela & 1,1 & 1,5 & 1,2 & 0,7 & 1,0 \\
\hline Parda & 62,2 & 63,7 & 63,2 & 57,8 & 62,0 \\
\hline Indígena & 0,5 & 0,6 & 0,2 & 0,1 & 0,7 \\
\hline Não informada & 0,0 & - & 0,0 & 0,0 & 0,0 \\
\hline $\begin{array}{l}\text { Proporção da população } \\
\text { com baixa renda }\end{array}$ & 64,9 & 62,9 & 63,2 & 58,2 & 58,9 \\
\hline Taxa de analfabetismo & 23,0 & 21,2 & 20,1 & 21,9 & 20,4 \\
\hline Taxa de desemprego & 9,7 & 9,0 & 9,2 & 8,0 & 11,3 \\
\hline $\mathrm{IDH}^{*}$ & 0,593 & 0,603 & 0,626 & 0,625 & 0,619 \\
\hline
\end{tabular}

Informação referente ao ano de 2000. 


\begin{tabular}{|c|c|c|c|c|c|c|}
\hline \multirow[t]{32}{*}{$\begin{array}{l}\vec{J} \\
\tilde{J} \\
0 \\
\stackrel{0}{\tilde{N}} \\
\tilde{J} \\
\tilde{D}\end{array}$} & $\begin{array}{l}\text { Tabela 2. Distribuição (núr } \\
\text { das vítimas e do evento. B }\end{array}$ & $\begin{array}{l}\text { e proporç } \\
\text { Brasil, } 19\end{array}$ & $\begin{array}{l}\text { o) } \operatorname{dos} c \\
6-2010 .\end{array}$ & s por homicídio (X85-Y09) segu & aracterísti & \\
\hline & \multirow[t]{2}{*}{ Variáveis } & \multirow[t]{2}{*}{ Casos } & \multirow[t]{2}{*}{$\%$} & \multirow{2}{*}{$\begin{array}{l}\text { Variáveis } \\
\text { Faixa etária }\end{array}$} & \multirow[t]{2}{*}{ Casos } & \multirow[t]{2}{*}{$\%$} \\
\hline & & & & & & \\
\hline & Masculino & 37.644 & 92,4 & 0 a 9 anos & 284 & 0,7 \\
\hline & Feminino & 3.059 & 7,5 & 10 a 14 anos & 475 & 1,2 \\
\hline & Ignorado & 51 & 0,1 & 15 a 19 anos & 6.460 & 15,9 \\
\hline & Não informado & 2 & 0,0 & 20 a 29 anos & 17.485 & 42,9 \\
\hline & Escolaridade & & & 30 a 39 anos & 7.821 & 19,2 \\
\hline & Nenhuma & 2.842 & 7,0 & 40 a 49 anos & 3.780 & 9,3 \\
\hline & 1 a 3 anos & 8.648 & 21,2 & 50 a 59 anos & 1.751 & 4,3 \\
\hline & 4 a 7 anos & 11.681 & 28,7 & 60 e mais & 1.386 & 3,4 \\
\hline & 8 a 11 anos & 3.657 & 9,0 & Não informada & 348 & 0,9 \\
\hline & 12 e mais & 480 & 1,2 & Ignorada & 966 & 2,4 \\
\hline & Não informado & 9.386 & 23,0 & Macrorregião de residência & & \\
\hline & Ignorado & 4.062 & 10,0 & Centro-Leste & 3.079 & 7,6 \\
\hline & Estado civil & & & Centro-Norte & 1.057 & 2,6 \\
\hline & Solteiro & 30.857 & 75,7 & Extremo Sul & 3.185 & 7,8 \\
\hline & Casado & 4.408 & 10,8 & Leste & 18.612 & 45,7 \\
\hline & Viúvo & 295 & 0,7 & Nordeste & 1.861 & 4,6 \\
\hline & Separado judicialmente & 374 & 0,9 & Norte & 3.036 & 7,4 \\
\hline & União consensual & 89 & 0,2 & Oeste & 574 & 1,4 \\
\hline & Não informado & 2.895 & 7,1 & Sudoeste & 2.721 & 6,7 \\
\hline & Ignorado & 1.838 & 4,5 & Sul & 5.589 & 13,7 \\
\hline & Raça/cor & & & Ignorado & 1.042 & 2,6 \\
\hline & Branca & 2.440 & 6,0 & Local de Ocorrência do óbito & & \\
\hline & Preta & 4.095 & 10,0 & Hospital & 12.465 & 30,6 \\
\hline & Amarela & 55 & 0,1 & Estabelecimentos de saúde & 366 & 0,9 \\
\hline & Parda & 25.681 & 63,0 & Domicílio & 4.414 & 10,8 \\
\hline & Indígena & 37 & 0,1 & Via pública & 16.579 & 40,7 \\
\hline & Não informada & 8.448 & 20,7 & Outros & 5.584 & 13,7 \\
\hline & & & & Não informado & 668 & 1,6 \\
\hline & & & & Ignorado & 680 & 1,7 \\
\hline & & & & TOTAL & 40.756 & 100,0 \\
\hline
\end{tabular}

acentuados foram observados nas faixas de 15 a 19 anos $(312,2 \%)$ e de 10 a $14(219,4 \%)$. Vale salientar que a partir de 2007, a faixa de 15 a 19 anos tornou-se a segunda faixa etária com maior risco absoluto, ultrapassando o grupo dos 30 a 39 anos. O grupo etário dos 20 aos 29 anos foi o que apresentou as maiores taxas brutas em todos os anos, com um incremento de $181,8 \%$.

Na Tabela 4 é apresentada a TMH padronizada por idade, segundo a macrorregião de saúde, nela destacam-se as MRS Extremo Sul, Leste, Norte e Sul, com TMH superiores às taxas totais da Bahia em quase todo o período. O Extremo Sul passou do segundo para o primeiro lugar no ranking do Estado, com TMH de 22,3 em 1996, que atingiu 64,6 por 100.000 habitantes em 2010. A MRS Oeste aparece com as TMH mais baixas, em todos os anos. Ademais, foi a única região a apresentar queda das taxas entre 1996 e 2010 , evidenciada por variação negativa de $36,2 \%$.

A evolução da TMH na população dos 15 aos 39 anos, segundo macrorregião e sexo nos anos de 1996, 2003 e 2010, mostra que nesse período todas as MRS apresentaram incrementos proporcionais positivos das taxas de mortalidade totais, com exceção da MRS Oeste, onde foi observada uma redução de 21,2\% (Tabela 5). Quanto ao sexo feminino, destaca-se no ano de 2010, a TMH no Extremo Sul (22,0 por 100.000 mulheres), com uma variação percentual superior a 400\%. Já no sexo masculino, a TMH mais elevada foi na região Leste, também no ano de 2010, com 226,6 homicídios por 100.000 homens. Entre eles, a taxa de mortalidade da MRS Leste, 
Tabela 3. Evolução da taxa de mortalidade por homicídio e variação percentual ( $\Delta \%)$, segundo faixa etária. Bahia, 1996 a 2010.

\begin{tabular}{|c|c|c|c|c|c|c|c|c|}
\hline & \multicolumn{8}{|c|}{ Taxa de mortalidade por 100.000 habitantes } \\
\hline & 1996 & 1997 & 1998 & 1999 & 2000 & 2001 & 2002 & 2003 \\
\hline \multicolumn{9}{|l|}{ Faixa etária (anos) } \\
\hline 0 a 9 & 0,5 & 0,7 & 0,4 & 0,4 & 0,6 & 0,8 & 0,6 & 0,7 \\
\hline 10 a 14 & 2,0 & 1,6 & 1,0 & 0,5 & 1,0 & 1,3 & 0,9 & 1,3 \\
\hline 15 a 19 & 19,2 & 21,6 & 11,4 & 8,7 & 11,0 & 14,5 & 17,1 & 20,3 \\
\hline 20 a 29 & 32,9 & 34,8 & 21,4 & 14,7 & 21,0 & 27,6 & 31,0 & 38,9 \\
\hline 30 a 39 & 23,4 & 23,0 & 15,5 & 10,4 & 15,5 & 19,0 & 18,1 & 23,6 \\
\hline 40 a 49 & 16,9 & 16,6 & 13,0 & 9,3 & 10,8 & 14,2 & 12,5 & 12,9 \\
\hline 50 a 59 & 9,2 & 10,7 & 6,1 & 4,4 & 7,6 & 8,2 & 10,5 & 12,4 \\
\hline 60 e mais & 6,0 & 8,6 & 6,0 & 3,7 & 5,5 & 5,8 & 6,9 & 7,3 \\
\hline \multirow[t]{3}{*}{ Mortalidade total padronizada } & 14,8 & 15,6 & 9,9 & 6,9 & 9,7 & 12,2 & 13,0 & 15,8 \\
\hline & \multicolumn{7}{|c|}{ Taxa de mortalidade por 100.000 habitantes } & \\
\hline & 2004 & 2005 & 2006 & 2007 & 2008 & 2009 & 2010 & $\Delta \%^{*}$ \\
\hline \multicolumn{9}{|l|}{ Faixa etária (anos) } \\
\hline 0 a 9 & 0,7 & 0,8 & 0,6 & 1,0 & 0,5 & 0,8 & 1,3 & 136,6 \\
\hline 10 a 14 & 1,0 & 1,7 & 1,1 & 2,5 & 5,4 & 5,4 & 6,5 & 219,4 \\
\hline 15 a 19 & 19,2 & 23,8 & 29,5 & 37,7 & 51,5 & 73,8 & 79,3 & 312,2 \\
\hline 20 a 29 & 40,6 & 50,2 & 56,6 & 56,8 & 77,2 & 82,1 & 92,8 & 181,8 \\
\hline 30 a 39 & 23,7 & 28,7 & 34,0 & 34,9 & 39,9 & 43,2 & 52,1 & 122,7 \\
\hline 40 a 49 & 17,7 & 19,7 & 22,3 & 21,6 & 24,5 & 26,7 & 27,3 & 61,9 \\
\hline 50 a 59 & 12,6 & 14,8 & 17,4 & 15,2 & 17,4 & 15,4 & 18,2 & 98,6 \\
\hline 60 e mais & 7,5 & 9,1 & 9,9 & 9,9 & 9,4 & 10,4 & 11,6 & 94,3 \\
\hline Mortalidade total padronizada & 16,5 & 20,1 & 23,1 & 24,1 & 30,6 & 34,5 & 38,9 & 163,8 \\
\hline
\end{tabular}

*variação percentual.

reduziu de 97,6 por 100.000 em 1996 , para 86,0 por 100.000 em 2003, mas voltou a ascender em 2010, contribuindo para que esta região atingisse a maior TMH entre os homens na faixa etária considerada.

\section{Discussão}

O perfil de mortalidade por homicídio na Bahia, apresentado nos resultados, assemelha-se ao padrão de outras regiões do Brasil. A sobremortalidade masculina, de maioria jovem, com pouca escolaridade, predominantemente negra, já faz parte do paradigma dos homicídios, no país e no mundo $0^{8,9,28-31}$.

Um destaque preocupante, observado no presente estudo, foi a variação dos homicídios na população infanto-juvenil. Esse grupo apresentou a maior elevação da mortalidade por homicídio na Bahia, quando analisada a evolução das taxas, por faixa etária durante o período de 1996 a 2010 .
Embora não se possa afirmar com precisão a existência de um processo de infantilização e juvenilização no panorama dos homicídios no Estado, devido à limitação relacionada ao pequeno tamanho da série histórica. É bem provável que esta seja uma característica "alarmante" no diagnóstico da mortalidade por homicídio, o qual esse estudo pretende subsidiar.

Enfatiza-se que, além do risco individual e dos comportamentos de risco, existem vulnerabilidades que estão relacionadas ao território. Uma evidência que reflete os diversos contextos onde os homicídios ocorreram, é a diferença na distribuição das TMH entre as nove macrorregiões de saúde da Bahia.

Fica claro que houve um incremento nas TMH em praticamente todas as MRS, principalmente entre os homens de 15 a 39 anos de idade. Mas como explicar essas diferenças entre as regiões? E quais hipóteses surgem destas evidências?

Primeiro é preciso ponderar os incrementos encontrados nas TMH, tendo por base a qualidade da informação. Entretanto, a melhoria na 


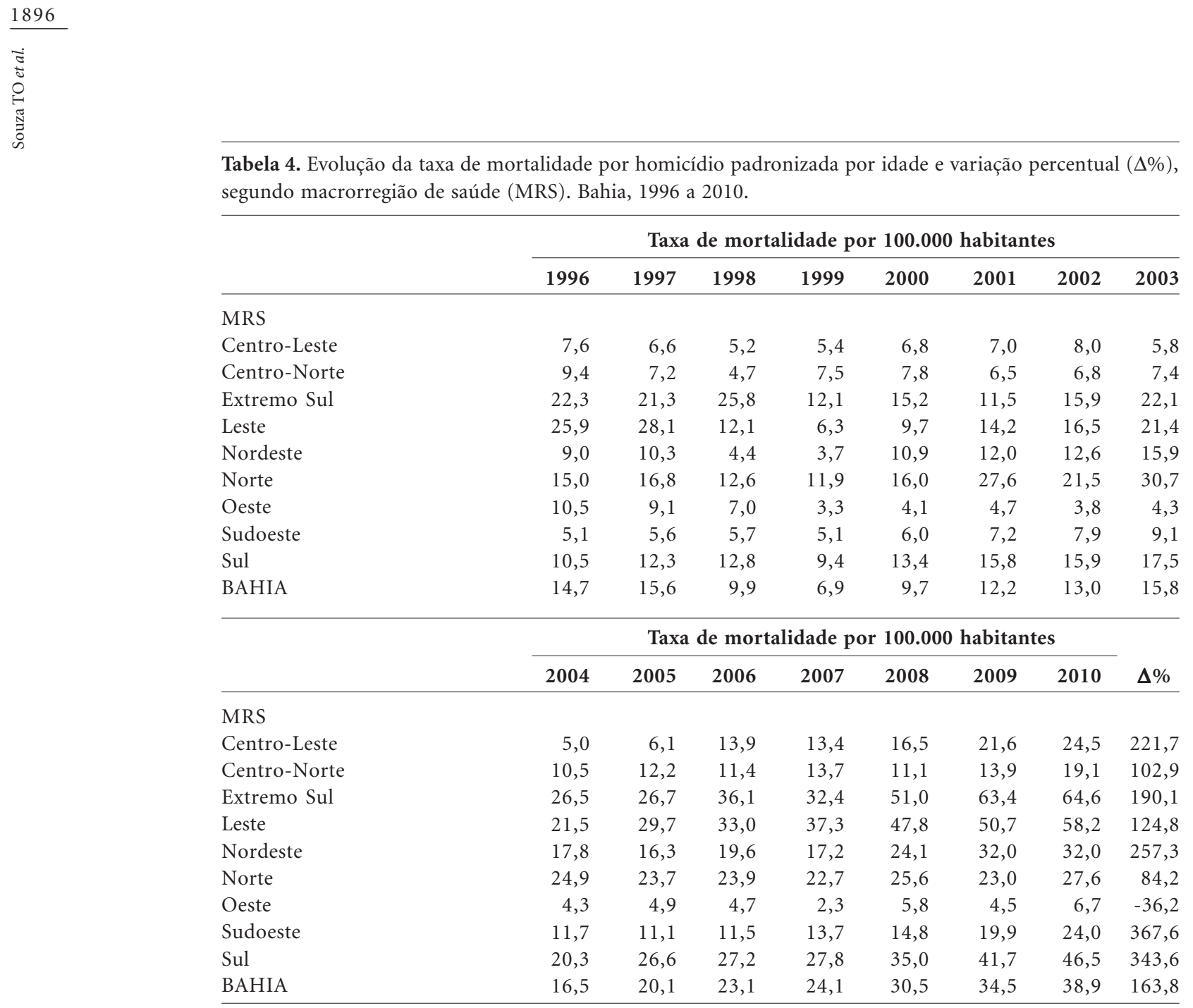

cobertura dos óbitos e qualidade das informações acerca da causa, não dão conta de explicar as variações entre as taxas de homicídio. Em seguida, atentar que a evolução da mortalidade por homicídios entre as MRS deve ser considerada com cautela, pois assim como é difícil explicar a queda nas TMH em alguns Estados, como é o caso de São Paulo, também são escassos os estudos que abordam o incremento das TMH nas diversas regiões e municípios, como é o caso da Bahia $^{32,33}$.

Alguns autores, afirmam que as variações nos índices de homicídio, na Bahia e em outras regiões, estão relacionadas à densidade demográfi$\mathrm{ca}$, ao índice de trabalho e renda, aos investimentos na pasta da saúde e segurança pública e às taxas de encarceramento-aprisionamento, dentre outros determinantes sociais da saúde $\mathrm{e}^{32-34}$.

A questão chave na abordagem por MRS, sustentada aqui, é a hipótese de que a "epidemia do homicídio" não está restrita as regiões metropolitanas; tampouco a fatores socioeconômicos isolados. Atualmente, os estudos apontam para a disseminação e o espalhamento dos homicídios para outras regiões e, por isso, essas áreas precisam de uma maior atenção por parte das autoridades e pesquisadores do tema ${ }^{11,33}$.

As MRS onde se concentraram as maiores TMH, no presente trabalho, são as do Extremo Sul, Sul, Leste e Norte. Essas regiões estão localizadas no litoral do Estado, e fazem fronteira ao Sul com o Estado do Espírito Santo e ao Norte com os Estados de Alagoas e Pernambuco, justamente os Estados que ocupam a segunda, a primeira e a quarta posição no ordenamento das unidades federativas por taxa de homicídio em 100.000 no Brasil em $2010^{11}$.

Provavelmente, a proporção de jovens na população, a densidade populacional, a taxa de desemprego e os territórios de fronteira, ajudam a explicar as elevadas taxas de homicídio nessas MRS. Com relação às altas taxas de homicídio em áreas fronteiriças, é possível que os corredores de ligação (rodovias, estradas e municípios 
Tabela 5. Evolução da taxa bruta de mortalidade por homicídio, dos 15 aos 39 anos, e variação percentual $(\Delta \%)$, segundo macrorregião de saúde e sexo. Bahia, 1996, 2003 e 2010.

\begin{tabular}{|c|c|c|c|c|c|c|}
\hline \multirow{3}{*}{$\begin{array}{l}\text { Macrorregião } \\
\text { de Saúde }\end{array}$} & \multicolumn{6}{|c|}{ Taxa de mortalidade por $\mathbf{1 0 0 . 0 0 0}$ habitantes } \\
\hline & \multicolumn{3}{|c|}{1996} & \multicolumn{3}{|c|}{2003} \\
\hline & Masculino & Feminino & Total & Masculino & Feminino & Total \\
\hline Centro-Leste & 19,8 & 4,3 & 11,9 & 17,9 & 1,4 & 9,7 \\
\hline Centro-Norte & 24,9 & 1,3 & 13,2 & 18,7 & 4,7 & 11,8 \\
\hline Extremo-Sul & 58,3 & 4,0 & 31,4 & 66,8 & 2,0 & 34,7 \\
\hline Leste & 97,6 & 6,2 & 49,7 & 86,0 & 3,3 & 43,0 \\
\hline Nordeste & 25,3 & 2,2 & 13,7 & 47,6 & 3,1 & 25,4 \\
\hline Norte & 47,6 & 5,8 & 26,6 & 98,5 & 5,6 & 52,5 \\
\hline Oeste & 24,4 & 2,9 & 14,0 & 13,0 & 1,3 & 7,3 \\
\hline Sudoeste & 13,0 & 2,4 & 7,7 & 31,1 & 1,9 & 16,6 \\
\hline Sul & 32,7 & 3,3 & 17,9 & 54,6 & 6,2 & 30,5 \\
\hline BAHIA & 48,6 & 4,3 & 26,1 & 55,1 & 3,3 & 29,0 \\
\hline \multirow{3}{*}{$\begin{array}{l}\text { Macrorregião } \\
\text { de Saúde }\end{array}$} & \multicolumn{6}{|c|}{ Taxa de mortalidade por 100.000 habitantes } \\
\hline & \multicolumn{3}{|c|}{2010} & \multicolumn{3}{|c|}{$\Delta \%^{*}$} \\
\hline & Masculino & Feminino & Total & Masculino & Feminino & Total \\
\hline Centro-Leste & 92,1 & 5,5 & 48,3 & 365,7 & 27,7 & 305,8 \\
\hline Centro-Norte & 60,2 & 5,1 & 32,9 & 141,2 & 282,4 & 149,5 \\
\hline Extremo-Sul & 214,1 & 22,0 & 117,6 & 267,0 & 444,3 & 275,2 \\
\hline Leste & 226,6 & 13,6 & 116,0 & 132,1 & 118,2 & 133,4 \\
\hline Nordeste & 109,3 & 9,9 & 59,5 & 332,0 & 356,2 & 334,1 \\
\hline Norte & 91,8 & 4,7 & 48,3 & 92,6 & $-18,2$ & 81,5 \\
\hline Oeste & 19,5 & 2,2 & 11,1 & $-20,1$ & $-25,6$ & $-21,2$ \\
\hline Sudoeste & 83,5 & 5,3 & 44,5 & 541,1 & 120,9 & 480,0 \\
\hline Sul & 163,1 & 11,1 & 86,5 & 398,0 & 231,7 & 381,8 \\
\hline BAHIA & 143,0 & 9,7 & 75,5 & 194,3 & 125,2 & 189,6 \\
\hline
\end{tabular}

* variação percentual das taxas de mortalidade por sexo entre os anos de 1996 e 2010.

contíguos) entre as MRS e os Estados, favoreçam o espalhamento e a interiorização dos homicídios $^{8,35}$.

Em estudo recente, Sousa ${ }^{33}$ identificou dois conglomerados de homicídios na Bahia, um no entorno de Salvador (situado na MRS Leste) e outro entre os municípios de Porto Seguro e Itabuna (nas MRS Extremo Sul e Sul, respectivamente). Esses achados corroboram o que foi observado no presente estudo, indicando que essas áreas apresentam dinâmicas propiciadoras de homicídio.

De modo semelhante, a taxa de desemprego foi mais elevada nas regiões com as maiores taxas de homicídio e as maiores variações percentuais nas TMH. Isso reforça a hipótese da importância das características socioeconômicas como fator explicativo para a elevação dos homicídios $^{17,33,34}$.
Peres et al. ${ }^{34}$ afirmam que a expansão econômica, com o aumento no número de empregos formais e o investimento em políticas sociais, levam a melhores condições de vida, principalmente para os jovens, prevenindo o seu envolvimento com o tráfico de drogas ou outras atividades ilícitas.

Em relação ao Índice de Desenvolvimento Humano das Macrorregiões (IDH) e as outras características socioeconômicas, é importante entender que a ocorrência dos homicídios não se explica a partir de fatores analisados de forma independente dos contextos ambientais. Por exemplo, os melhores valores no IDH foram encontrados nas regiões Leste e Extremo Sul, exatamente onde ocorreram as maiores TMH.

A explicação para esse aparente paradoxo é que nem sempre as regiões mais abastadas, apresentam menor nível de violência. Pelo contrário, quanto maior o tamanho da população e suas 
riquezas, maior a importância da concentração de renda e, consequentemente, da desigualdade socia ${ }^{35,36}$. Portanto, o maior risco de algumas MRS em comparação a outras, parece não estar relacionado à pobreza ou a qualquer outro fator socioeconômico isolado. De modo mais coerente, acredita-se que as variações nas TMH estejam relacionadas, principalmente, ao grau de atendimento das necessidades básicas de cada população $0^{36}$.

Desse modo, se os óbitos são considerados como um importante indicador de saúde, e o homicídio indicador universal dos níveis de violência, pode-se afirmar que as MRS do Extremo Sul, Leste, Norte e Sul, encontram-se mais vulneráveis em relação às demais, sendo necessário identificar de que forma as desigualdades sociais nelas existentes se relacionam aos homicídios.

\section{Considerações Finais}

A análise e o enfrentamento dos homicídios a partir de realidades locorregionais, considerando-se suas características socioeconômicas e culturais, parece ser um caminho possível para avançar no conhecimento desse grave problema social que vem afetando diretamente a saúde da população do país e nele atuar por meio de estratégias e planejamento de ações intersetoriais.

No entanto, é preciso avaliar a qualidade da informação que embasa as análises, sobretudo no tocante à causa básica do óbito, fator que tem um peso importante e limitante nas inferências que possam ser feitas.

Investigar a relação entre a melhoria da assistência à saúde e o nível de letalidade das vio- lências é fundamental para incentivar os investimentos na atenção prestada às vítimas, mas também na prevenção desses eventos. As grandes regiões, com maior número de habitantes, também são as que costumam apresentar maior capacidade instalada de serviços de saúde em geral, e particularmente de serviços de urgência e emergência. É, pois, necessário investigar os investimentos feitos na pasta da saúde e a qualidade dos serviços de saúde existentes nas diversas regiões estudadas.

Em cada MRS encontram-se municípios polo, de maior poder econômico, que concentram a maioria dos serviços de saúde de alta complexidade, a maioria das vagas em instituições públicas e privadas de ensino superior e a maior parcela das oportunidades de emprego e renda. A combinação desses fatores, associada à alta densidade populacional dessas regiões, favorece o aparecimento de ambientes com elevados níveis de desorganização social, no qual a dificuldade na resolução de conflitos resulta em altas taxas de homicídio. A isso se alia a facilidade de obtenção de armas de fogo, com as quais grande parte dos homicídios são perpetrados no país ${ }^{3,5}$.

Sendo assim, espera-se que os resultados apoiem a elaboração de outros estudos sobre a influência de áreas fronteiriças no processo de interiorização e espalhamento dos homicídios, sobretudo nas regiões Norte e Nordeste do Brasil ${ }^{2,11}$. Além disso, que sirva de subsídio para a criação de políticas públicas capazes de envolver segurança pública, saúde e sociedade civil, com o propósito de trabalharem em conjunto em todas as dimensões que o enfrentamento da violência requer.

\section{Colaboradores}

TO Souza, ER Souza e LW Pinto participaram igualmente de todas as etapas de elaboração do artigo. 


\section{Referências}

1. Brasil. Ministério da Saúde (MS). Portaria MS/GM no 737 de 16 de maio de 2001. Política Nacional de Redução de Morbimortalidade por Acidentes e Violências. Diário Oficial da União 2001; 18 maio.

2. Dahlberg LL, Krug EG. Violência: um problema global de saúde pública. Cien Saude Colet 2007; 11(Supl.):1163-1178.

3. Brasil. Ministério da Saúde (MS). Secretaria de Vigilância em Saúde. Impacto da violência na saúde dos brasileiros. [site na Internet]. [acessado 2012 out 20]. Disponível em: http://portal.saude.gov.br/ portal/arquivos/pdf/impacto_violencia.pdf

4. Minayo MCS. Violência e Saúde. Rio de Janeiro: Editora Fiocruz; 2006.

5. Peres MFT, Cardia N, Santos PC. Homicídios de crianças e jovens no Brasil: 1980-2002. São Paulo: Núcleo de Estudos da Violência; 2006.

6. Souza ER. Homicídios no Brasil: o grande vilão da saúde pública na década de 80. Cad Saude Publica 1994; 10(Supl. 1):45-60.

7. Minayo MCS, Souza ER. Violence for All. Cad Saude Publica 1993; 9(1):65-78.

8. Souza ER, Lima MLC. Panorama da violência urbana no Brasil e suas capitais. Cien Saude Colet 2007; 11(Supl.):1211-1222.

9. Krug EG, Dahlberg LL, Mercy JA, Zwi AB, Lozano $\mathrm{R}$, editores. Relatório Mundial sobre violência e saúde. Genebra: OMS; 2002

10. Souza ER, Minayo MCS, Silva CMF, Reis AC, Malaquias JV, Veiga JPC, Santos NC. Borges KF, Silva CFR. Análise temporal da mortalidade por causas externas no Brasil: Décadas de 80 e 90. In: Minayo MCS, Souza ER, organizadoras. Violência sob o olhar da saúde: a infrapolitica da contemporaneidade brasileira. Rio de Janeiro: Fiocruz; 2003.

11. Waiselfisz JJ. Mapa da Violência 2011: Os Jovens do Brasil. São Paulo, Brasília: Instituto Sangari, Ministério da Justiça; 2011.

12. Viana LAC. Mortalidade por causas externas: evolução temporal das desigualdades sociais no espaço intra-urbano. [dissertação]. Salvador: Universidade Federal da Bahia; 2010.

13. Paim JS, Costa HOG, Vilasboas ALQ. Política pública e controle da violência: um estudo de caso na cidade de Salvador, Bahia, Brasil. Cad Saude Publica 2009; 25(3):485-494.

14. Araújo EM, Costa MCN, Hogan VK, Mota ELA, Araújo TM, Oliveira NF. Diferenciais de raça/cor da pele em anos potenciais de vida perdidos por causas externas. Rev Saude Publica 2009; 43(3):405-412.

15. Santos JEF. Homicídio entre jovens de uma periferia de Salvador, Bahia: um relato de experiência sobre a violência e o desenvolvimento humano. Rev bras crescimento desenvolv hum 2007; 17(3):72-83.

16. Nunes M, Paim JS. Um estudo etno-epidemiológico da violência urbana na cidade de Salvador, Bahia, Brasil: os atos de extermínio como objeto de análise. Cad Saude Publica 2005; 21(1):459-468.

17. Macedo AC, Paim JS, Silva LMV, Costa MCN. Violência e desigualdade social: mortalidade por homicídios e condições de vida em Salvador, Brasil. Rev Saude Publica 2001; 35(6):515-522.
8. Freitas ED, Paim JS, Silva LMV, Costa MCN. Evolução e distribuição espacial da mortalidade por causas externas em Salvador, Bahia, Brasil. Cad Saude Publica 2000; 16(4):1059-1070.

19. Costa AJL, Kale PL, Vermelho LL. Indicadores de Saúde. In: Medronho RA, editor-chefe. Epidemiologia. São Paulo: Editora Atheneu; 2009.

20. Araújo JD, Ferreira ESM, Nery GC. Regionalização dos serviços de saúde pública: a experiência do Estado da Bahia, Brasil. Rev Saude Publica 1973, 7(1):1-19.

21. Castro MSM, Silva BFA, Assunção RM, Filho CCB. Regionalização como estratégia para a definição de políticas públicas de controle de homicídios. Cad Saude Publica 2004; 20(5):1269-1280.

22. Organização Mundial de Saúde (OMS). Classificação estatística internacional de doenças e problemas relacionados à saúde. [site na Internet] Décima Revisão (CID-10). Centro Brasileiro de Classificação de Doenças. São Paulo: EDUSP, 2008. [acessado 2012 jun 01]. Disponível em: http://www.datasus. gov.br/cid10/v2008/cid10.htm

23. Departamento de Informática do SUS (Datasus). Informações de Saúde. [site na Internet] Brasil: MS [acessado 2012 mai 29]. Disponível em: http:// www2.datasus.gov.br/DATASUS/index.php?area $=02$

24. Instituto Brasileiro de Geografia e Estatística (IBGE). Censo demográfico 2010. [site na Internet] Brasil: 2012 [acessado 2012 dez 20]. Disponível em: http://www. ibge.gov.br/home/estatistica/populacao/censo2010/ default.shtm

25. Programa das Nações Unidas para o Desenvolvimento (PNUD). Ranking do IDH dos Municípios do Brasil. [site na Internet] Brasil: ONU; 2012 [acessado $2012 \mathrm{dez}$ 20]. Disponível em: http://www. pnud. org.br/atlas/ranking/IDH_Municipios_Brasil_2000 .aspx?indiceAccordion=1\&li=li_Ranking2003

26. Bahia Secretaria de Estado da Saúde. Plano Diretor de Regionalização. [site na Internet] Bahia; 2012 [acessado 2012 ago 11]. Disponível em: http://ftp.saude. ba.gov.br/int_estagio.html

27. World Health Organization (WHO). Age-standardized mortality rates (per 100000 population). [site na Internet] Geneva: World Health Organization; 2011 [acessado 2012 jun 01]. Disponível em: http://www. who.int/whosis/indicators/WHS2011_Indicator Compendium_20110530.pdf

28. Soares FAM. Vitimização por homicídios segundo características de raça no Brasil. Rev Saude Publica 2011; 45(4):745-755

29. Souza ER. Masculinidade e violência no Brasil: contribuições para a reflexão no campo da saúde. Cien Saude Colet 2005; 10(1):59-70

30. Peres MFT. Homicídios, risco e vulnerabilidade: para uma discussão da dinâmica da vitimização por homicídios. In: Cruz MVG, Batitucci EC, organizadores. Homicídios no Brasil. Rio de Janeiro: Editora FGV; 2007. 
31. Gawryszewski VP, Scarpelini S, Dib JA, Jorge MHPM, Pereira JGA, Morita M. Atendimentos de emergência por lesões decorrentes de causas externas: características das vítimas e local de ocorrência, Estado de São Paulo, Brasil, 2005. Cad Saude Publica 2008; 24(5):1121-1129.

32. Peres MFT, Vicentin D, Nery MB, Lima RS, Souza ER, Cerda M, Cardia N, Adorno S. Queda dos homicídios em São Paulo, Brasil: uma análise descritiva. Rev Panam Salud Publica 2011; 29(1):17-26.

33. Sousa CAM. Homicídios na Bahia em 2009: determinantes socioeconômicos e a mortalidade por violência [dissertação]. Rio de Janeiro: Escola Nacional de Saúde Pública Sergio Arouca; 2012.

34. Peres MFT, Almeida JF, Vicentin D, Cerda M, Cardia N, Adorno S. Queda dos homicídios no município de São Paulo: uma análise exploratória de possíveis condicionantes. Rev bras epidemiol 2011; 14(4):709-721.

35. Andrade SM, Soares DA, Souza RKT, Matsuo T, Souza HD. Homicídios de homens de quinze a 29 anos e fatores relacionados no estado do Paraná, de 2002 a 2004. Cien Saude Colet 2011; 16(Supl. 1):1281-1288.

36. Barata RB. Como e Por Que as Desigualdades Sociais Fazem Mal à Saúde. Rio de Janeiro: Editora Fiocruz; 2009.

Artigo apresentado em 22/04/2013

Aprovado em 06/08/2013

Versão final apresentada em 09/08/2013 